\title{
Direct Wave-Vector Excitation in an Indirect-Band-Gap Semiconductor of Silicon with an Optical Near-field
}

\author{
Masashi Noda, $,{ }^{1,},{ }^{*}$ Kenji Iida, ${ }^{1}$ Maiku Yamaguchi, ${ }^{2}$ Takashi Yatsui, ${ }^{2}$ and Katsuyuki Nobusada ${ }^{1}$ \\ ${ }^{1}$ Department of Theoretical and Computational Molecular Science, Institute for Molecular Science, Myodaiji, \\ Okazaki, 444-8585, Japan \\ ${ }^{2}$ Department of Electrical Engineering and Information Systems, Graduate School of Engineering, The University \\ of Tokyo, 7-3-1, Hongo, Bunkyo-ku, Tokyo, 113-8656, Japan
}

(Received 24 August 2018; revised manuscript received 24 January 2019; published 17 April 2019)

\begin{abstract}
In this work, our first-principles calculations reveal that direct wave-vector excitation (i.e., interband transitions between different wavenumbers without phonon assistance) can occur in the indirect-bandgap semiconductor silicon. The wave-vector excitation is successfully induced by irradiation of a silicon thin film with an optical near-field (ONF). As a result, the absorption-band-edge energy $E_{\text {edge }}$ shifts to a lower photon energy of $1.6 \mathrm{eV}$ for ONF excitation from $E_{\text {edge }}$ of $2.1 \mathrm{eV}$ for the conventional excitation by propagating far-field light. The direct wave-vector excitation is caused by the sufficiently large components of wave vectors inherent in the ONF, and thus does not require phonon assistance. For a realistic silicon system, it is clarified that the wave-vector excitations are determined by the energy difference between the valence and conduction bands and occur irrespective of the initial and final wave vectors.
\end{abstract}

DOI: 10.1103/PhysRevApplied.11.044053

\section{INTRODUCTION}

Silicon is the most-mature material used in semiconductor technology and has been undoubtedly leading the way in the growth of the semiconductor industry. Electronic devices such as transistors, resistors, or a set of these components (i.e., integrated circuits), are largely based on silicon technology. In addition, silicon semiconductors have been widely used as a key ingredient material in optoelectronic devices such as solar cells. However, in comparison with the sufficiently high potentiality of silicon semiconductors in electronic devices, silicon semiconductors have an essential drawback when used in optoelectronic devices because of the low optical absorption and emission efficiency of silicon. Thus, the thickness of a silicon solar cell is typically on the order of hundreds of microns; this results in low solar-cell efficiency due to the high recombination efficiency and large thermal relaxation. In addition, there are no practical light-emitting devices made of silicon, such as light-emitting diodes. All these drawbacks originate entirely from the indirect-band-gap structure of

\footnotetext{
*noda@ccs.tsukuba.ac.jp
}

†Present address: University of Tsukuba

Published by the American Physical Society under the terms of the Creative Commons Attribution 4.0 International license. Further distribution of this work must maintain attribution to the author(s) and the published article's title, journal citation, and DOI. silicon. In indirect-band-gap semiconductors, the top of the valence band and the bottom of the conduction band have different wave vectors $(\mathbf{k})$. The wave-vector conservation law must be satisfied through an interband transition. Thus, visible light cannot directly cause an indirect interband transition, because the wave vector of visible light is 3 orders of magnitude smaller than the difference in the wave vectors of the indirect-band-gap structure. To solve the problem, phonon assistance, (i.e., electron-phonon coupling) is required for an interband transition between the different wave vectors. As a result of the indirect interband transition, the optical absorption and emission efficiency are very low.

Various practical approaches have been proposed to improve the optical properties of silicon semiconductors. Tandem structures of silicon and other materials, heterogeneous-material-doped silicon substrates, or specific structural designs of silicon substrates are typical examples [1-3]. Recently, an electric field enhancement due to plasmonic materials inside a silicon semiconductor was used to induce an interband transition in silicon [4-13]. These approaches should provide workable solutions to the low optical efficiency of silicon semiconductors. However, they still require phonon assistance, and are therefore not direct solutions to the problem of inducing interband transitions in indirect-band-gap silicon semiconductors.

In the present study, we propose a substantially different approach to induce interband transitions between 
different wave vectors in a silicon semiconductor. The concept is based on direct wave-vector excitation by large wave-vector components of an optical near-field (ONF). The physical mechanism underlying the concept is the fact that the ONF has wave vectors that are 3 or more orders of magnitude larger than those of incident propagating far-field (FF) light in the visible range. This is a natural consequence of spatially localized ONF in a nanostructure, according to the uncertainty principle. The physical concept of wave-vector excitation was proposed by Kirkengen and Bergli [14] in a simple mathematical model. The present authors demonstrated that direct wavevector excitation actually occurred in an one-dimensional Kronig-Penny model, which is physically analytical [15]. However, since all these theoretical studies are based on simple mode systems, there is a very large gap between the physical concept of direct wave-vector excitation and its actual realization in silicon nanotechnology. Therefore, we perform first-principles calculations of direct wave-vector excitation in a realistic silicon semiconductor system and verify the realization of a highly optically functional material made of silicon.

\section{METHODS}

\section{A. Theoretical formula}

Our theoretical method is based on a real-time and real-space grid-based time-dependent density-functionaltheory (DFT) approach developed in our group [16-18]. The time evolution of density in an interacting $N$-electron system is described by the time-dependent Kohn-Sham equation for auxiliary electronic wave functions $\psi_{j k}(\mathbf{r}, t)$ in the Coulomb gauge:

$$
\begin{aligned}
i \hbar \frac{\partial}{\partial t} \psi_{j k}(\mathbf{r}, t)= & {\left[-\frac{\hbar^{2}}{2 m} \nabla^{2}-i \hbar \frac{e}{m} \mathbf{A}(t) \cdot \nabla+\frac{e^{2}}{2 m} \mathbf{A}^{2}(t)\right.} \\
& \left.+V_{H}(\mathbf{r}, t)+V_{\mathrm{xc}}(\mathbf{r}, t)+V_{\mathrm{ion}}(\mathbf{r}, t)\right] \psi_{j k}(\mathbf{r}, t),
\end{aligned}
$$

where $m$ is the electron mass, $\mathbf{A}$ is the vector potential of an external field, $V_{H}$ is the Hartree potential, $V_{\mathrm{XC}}$ is the exchange-correlation (XC) potential, and $V_{\text {ion }}$ is the ionic pseudopotential. In the calculation of the ONF excitation, instead of using $\mathbf{A}$, we use the potentials given by Eq. (5) in the right-hand side of Eq. (1). The Hartree potential is obtained from the Poisson equation:

$$
\nabla^{2} V_{H}(\mathbf{r}, t)=-4 \pi e \rho(\mathbf{r}, t),
$$

where $e$ is the elementary charge and $\rho$ is the electron density given by

$$
\rho(\mathbf{r}, t)=\frac{2}{k_{\mathrm{tot}}} \sum_{j, k(\in \mathrm{occ})}\left|\psi_{j k}(\mathbf{r}, t)\right|^{2} .
$$

In Eq. (3), $k_{\text {tot }}$ is the total number of $k$-point grids, and the sum is performed for the occupied orbitals. For the $\mathrm{XC}$ potential, we use the adiabatic local-density approximation (LDA) with the ground-state LDA XC potential given by Perdew and Zunger [19]. We confirm that the results obtained with the LDA potential are almost the same as those obtained with the Perdew-Burke-Ernzerhof (PBE) potential [20]. Although a further investigation of the dependence on DFT functionals would be beneficial, it is very difficult to use a more-sophisticated DFT functional. This is because first-principles calculations of the ONF excitation have an enormous computational cost to treat a huge supercell. Thus, we focus here on the interactions between a realistic silicon system and the ONF, which inherently has large wave-vector components. For $V_{\text {ion, }}$, we use norm-conserving pseudopotentials with the Kleinman-Bylander separable form [21,22].

In the simulation of FF excitations, we can apply the standard Bloch condition, $\psi_{j k}(\mathbf{r})=\exp (i \mathbf{k} \cdot \mathbf{r}) u_{j k}(\mathbf{r})$, with $u_{j k}(\mathbf{r}+\mathbf{l})=u_{j k}(\mathbf{r})$, where $\mathbf{l}$ is one of the lattice vectors. Then we follow the electron dynamics of $u_{j k}(\mathbf{r}, t)$ instead of $\psi_{j k}(\mathbf{r}, t)$ using the vector potential $\mathbf{A}$ [see Eq. (1)] given as $\mathbf{A}(t)=A_{0} \hat{\mathbf{u}} \theta(t)$, where $\hat{\mathbf{u}}$ is a unit vector and $\theta$ is a step function. Detailed explanations of the calculation of the dielectric function are provided elsewhere [23].

Since wave-vector excitations are not taken into account under the Bloch condition, to calculate the ONF excitation, we use a supercell method by adopting the Born-von Karman periodic boundary condition [24,25]. The number of unit cells included in the supercell is then the same as that of the $k$-point grids in the calculation of the ground state. Since the standard approach of calculating a dielectric function [23] cannot be applied to ONF excitations, we define their absorption intensities by

$$
P \equiv \sum_{j_{c}, j_{v}, k_{c}, k_{v}} \int d \mathbf{r}\left|\psi_{j_{c} k_{c}}^{*}(\mathbf{r}, 0) \psi_{j_{v} k_{v}}(\mathbf{r}, t)\right|^{2}
$$

where subscripts $c$ and $v$ denote the conduction and valence bands, respectively. In Eq. (4), the overlap integral of wave functions squared corresponds to the total change in the electron occupation of Bloch states (i.e., the transition probability). It is also noted that by calculating the FF excitation intensity with Eq. (4), we can reasonably compare the intensities of the ONF and FF excitations.

\section{B. Computational details}

The geometrical optimization is performed with the QUANTUM ESPRESSO program package with the PBE functional and a cutoff of 30 Ry $[20,26]$. We use $12 \times 12 \times$ $1 \Gamma$-centered grids for $k$-point sampling. The LDA functional is used hereafter because the results with the LDA and PBE functionals are qualitatively identical. It is known that the LDA and PBE functionals often underestimate 
band-gap energies [27]. However, the focus of this study is not to quantitatively evaluate band-gap energies but is to demonstrate wave-vector excitation in realistic systems. In fact, the indirect-band-gap structure is obtained with the functionals used. Thus, it is reasonably considered that the choice of an exchange-correlation function has, at least, a negligible influence on the discussion in this study.

Figures 1(a)-1(c) show the primitive cell of the three silicon bilayers and its band diagram, from which a typical indirect-band-gap structure is clearly recognized. The minimum band-gap energy between the top of the valence band and the bottom of the conduction band is $0.3 \mathrm{eV}$ [the red arrow in Fig. 1(c)], where their wave vectors are different from each other. The vertical energy difference at the $\Gamma$ point [the black arrow in Fig. 1(c)] is $2.0 \mathrm{eV}$, which is 1.7 $\mathrm{eV}$ higher than the minimum band-gap energy.

The optimized geometry is used for the calculations with SALMON (SCALABLE AB-INITIO LIGHT-MATER SIMULATOR FOR OPTICS AND MANOSCIENCE), a program we developed. In the calculations, we choose the orthorhombic unit cell of the $\mathrm{Si}(111)$ surface [Figs. 2(a) and 2(b)], to which the $\Gamma$-centered $32 \times 16 \times 1$ constant grids are applied for the $k$-point sampling. This number of $k$-point grids is needed to obtain well-converged results for the absorption intensity. Although at first glance this system has a direct band-gap structure [see Fig. 2(c)], the difference in the band-gap structure in Fig. 2(c) from that in Fig. 1(c) is due to the band folding of the extended cell larger than the primitive cell [28]. For example, the $M$ point of the primitive cell is transferred to the $\Gamma$ point of the orthorhombic cell.
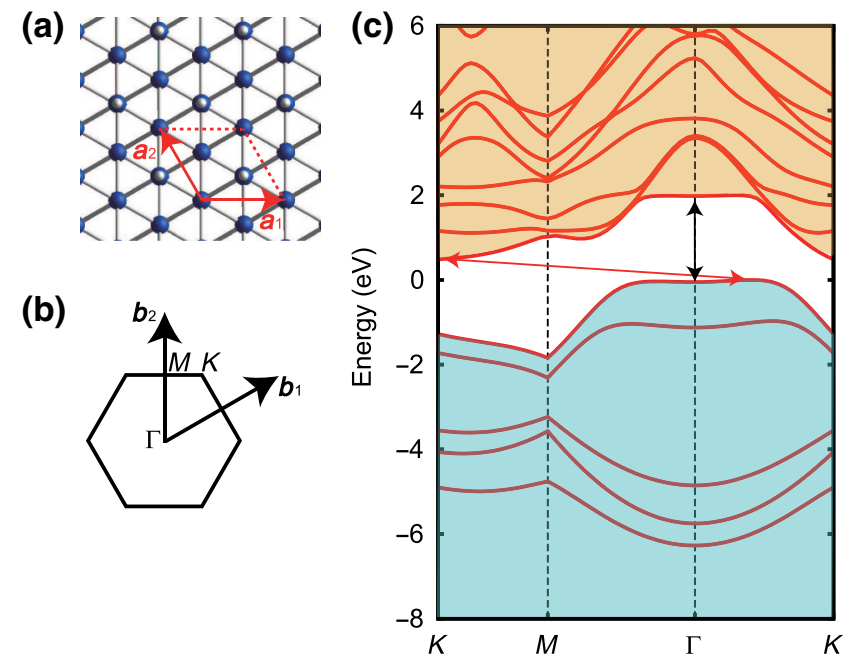

FIG. 1. (a) The top view of the Si(111) surface for the primitive cell of three silicon bilayers. Gray and blue circles represent silicon and hydrogen atoms, respectively. Vectors $\mathbf{a}_{1}$ and $\mathbf{a}_{2}$ are lattice vectors. (b) The reciprocal vectors $\mathbf{b}_{1}$ and $\mathbf{b}_{2}$ (black arrows) for the primitive cell. (c) Band diagram of the primitive cell of the three silicon bilayers. The black and red arrows denote the direct and indirect band gaps, respectively. (a)

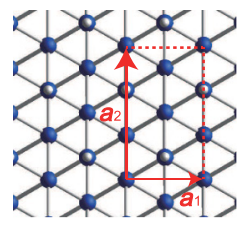

(b)

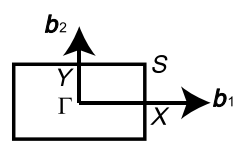

FIG. 2. (a) Top view of the orthorhombic unit cell of three silicon bilayers. (b) The reciprocal vectors. (c) Indirect-band-gap structure of silicon.

Thus, for far-field excitation, the lowest-energy transition at the $\Gamma$ point becomes zero due to the folded band structure. For all the calculations, we use orthorhombic grids with a constant mesh spacing of $0.32 \times 0.277 \times 0.333 \AA^{3}$ and a constant time step of $2.0 \times 10^{-3} \mathrm{fs}$.

The electric field of the laser pulse is set to $10^{9} \mathrm{~W} / \mathrm{cm}^{2}$ in accordance with the ONF used. The FF excitation intensity given by Eq. (4) increases infinitely as the computed cell size increases, while the ONF excitation intensity is almost proportional to the surface density of the ONF source. Thus, when Eq. (4) is used, we evaluate the FF excitation intensity per unit surface area of $2 \times 2 \mathrm{~nm}^{2}$ for comparison.

Because a huge supercell consisting of 8192 atoms is needed to calculate the present ONF excitation, it is essential to use a supercomputer for this study. The numerical calculations are mainly performed with the $\mathrm{K}$ computer at the RIKEN Advanced Institute for Computational Science. Each node consists of a SPARC64 VIIIfx processor (eight cores, $2.0 \mathrm{GHz}$ ) and $16 \mathrm{~GB}$ of memory. The most-timeconsuming part is the calculation of the electron dynamics under the ONF; the elapsed time for this is approximately $13 \mathrm{~h}$ when 4096 nodes are used.

\section{Silicon system irradiated by $\mathrm{ONF}$}

To study the ONF excitation in silicon, we use three silicon bilayers whose $\mathrm{Si}(111)$ surfaces are terminated by hydrogen atoms (Fig. 3). Silicon dangling bonds are eliminated here to retain the properties of bulk silicon. This three silicon-bilayer system is the largest one that we can treat because of the enormous computational cost to calculate the wave-vector excitation. Although it would be valuable to further investigate the dependence of the ONF excitation on the surface morphology [e.g., the (111) or (100) crystal face], which determines the band structure [29], quantitative investigation of the variation in the surface morphology is beyond the scope of this study. 


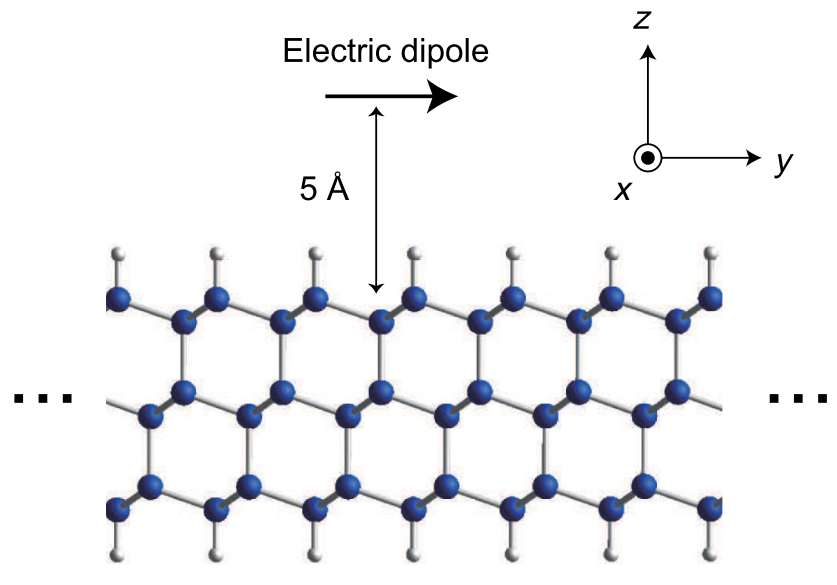

FIG. 3. Calculation model. Silicon bilayer, in which the Si(111) surfaces are terminated by hydrogen atoms. The electric dipole is put in a supercell.

In calculations of the ONF excitation, a $y$-polarized oscillating dipole as the source of the ONF is set at a distance of $0.5 \mathrm{~nm}$ from the top silicon layer. The potentials of the ONF are given by

$$
V_{\text {near }}(\mathbf{r}, t)=-\frac{\mathcal{M}_{0}\left(y-y_{p}\right)}{r^{3}} \sin (\omega t) \sin ^{2}\left(\frac{\pi t}{T}\right),
$$

where $\omega$ is the frequency of the oscillating dipole field and $\mathcal{M}_{0}$ is set to $3.76 \times 10^{-2} e \AA$, which generates a field intensity of $10^{9} \mathrm{~W} / \mathrm{cm}^{2}$ on the top silicon layer directly below the oscillating dipole. The density of the ONF source per surface area is then set to a sufficiently low value of $1 /\left(12 \times 11 \mathrm{~nm}^{2}\right)$, since we here focus on the interaction between the ONF source and the silicon film without interactions among the ONF sources. We set the pulse duration $T$ to $30 \mathrm{fs}$, and mainly discuss the result after the ONF is switched off to elucidate the mechanism of the excitation [30].

Because the magnitude of the ONF given by Eq. (5) is nonuniform in the spatial (r) domain, the ONF in the Fourier (k) domain includes large components of wave

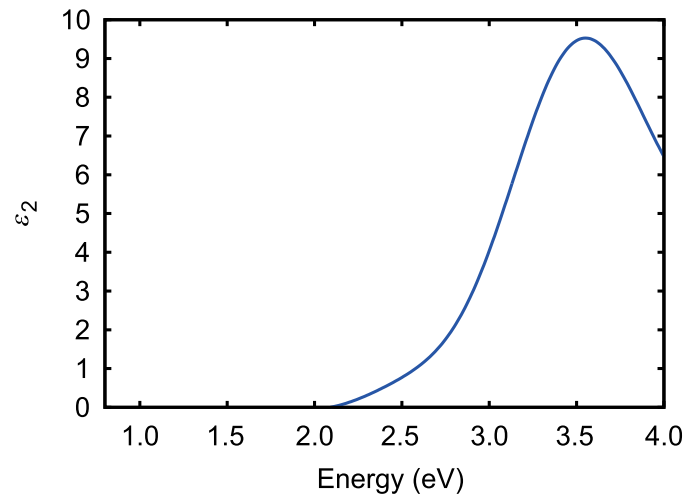

FIG. 5. Imaginary part of the dielectric function of the silicon thin film.

vectors as shown in Fig. 4. Therefore, the ONF can induce interband transitions between Bloch states with difference wave vectors (i.e., crystal momentum [24,31,32]). The total momentum of the electron system is unchanged because the ONF and the band structure have symmetric distributions in the $\mathbf{k}$ domain with respect to the origin $(\mathbf{k}=\mathbf{0})$.

\section{RESULTS AND DISCUSSION}

Before discussing the ONF excitation, we show the imaginary part of the dielectric function of the silicon thin film, corresponding to the conventional FF absorption spectrum in Fig. 5. The onset of the dielectric function (approximately $2.1 \mathrm{eV}$ ) is close to the vertical energy difference at the $\Gamma$ point, indicating simply that the electronic transitions occur between the valence and conduction bands within the same wave vector.

Figure 6(a) shows the absorption spectrum of the silicon thin film for ONF excitation. The absorption-band-edge energy for the ONF excitation is $1.6 \mathrm{eV}$, which is $0.5 \mathrm{eV}$ lower than that for the FF excitation. The electric field enhancement conventionally discussed in the plasmonics research field is useful only for increasing the absorption (a)

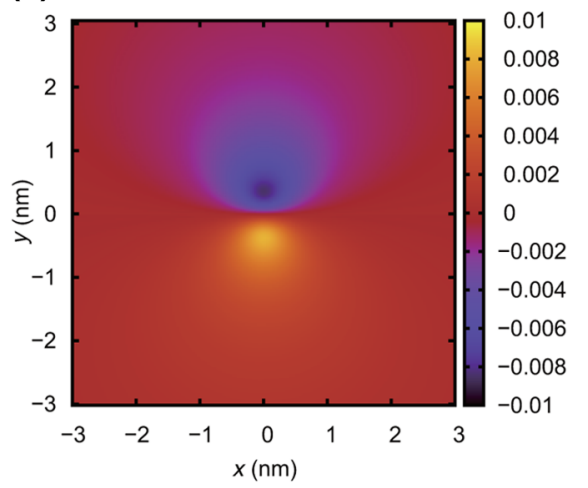

(b)

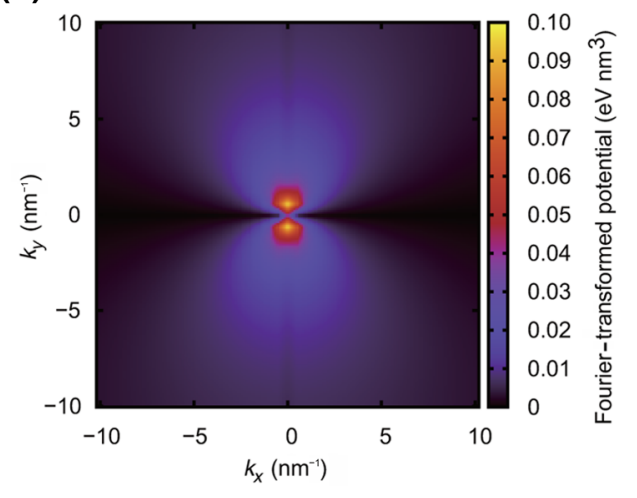

FIG. 4. (a) Potential of the ONF on the $x-y$ plane at a distance of $0.5 \mathrm{~nm}$ from the dipole and (b) its Fourier transform. 
(a) $\times 10^{-4}$

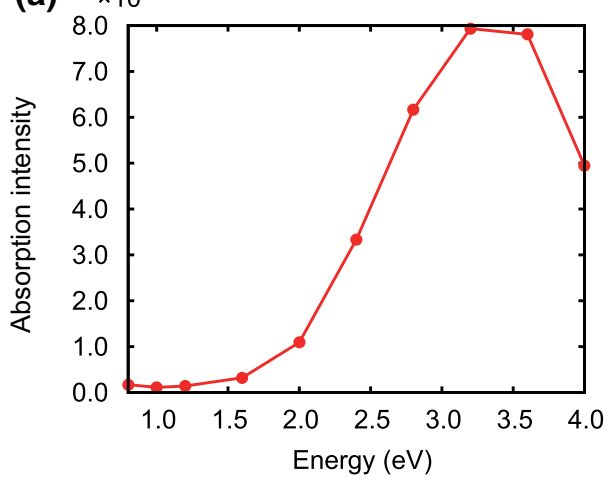

(b)

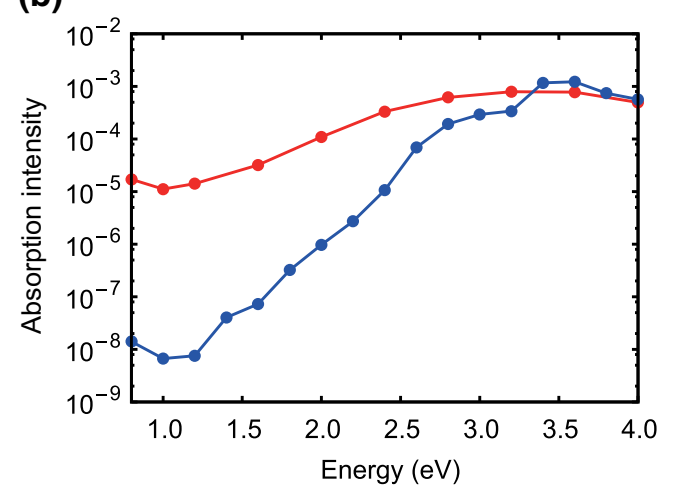

FIG. 6. (a) The absorption intensity for ONF excitation and (b) comparison between the absorption intensity for ONF excitation (red line and points) and $\mathrm{FF}$ excitation (blue line and points). The horizontal axis is the energy $(\mathrm{eV})$ of the incident external field. intensity; that is, this blueshift of the absorption edge is an intrinsic feature in the spatially localized field.

To identify the origins of the blueshift of the absorption spectrum, we examine the ONF excitation at $1.6 \mathrm{eV}$. Figure 7(a) shows a map of electronic excitation between the second-highest valence band and the lowest conduction band in the ONF excitation at $1.6 \mathrm{eV}$. The band structure directly corresponds to that in Fig. 2(c), whose the $X$ and $Y$ points are depicted below the energy surface. The interband transitions occur irrespective of the initial and final wave vectors if the excitation energy matches the frequency of the ONF [30]. Analogous wave-vector excitation is also found between the highest valence band and the lowest conduction band [30]. In Fig. 7(b), the absorption intensity of the excitation shown in Fig. 7(a) is plotted as a function of the variation in the wave vectors $\Delta k$ (equivalent to $\left|\mathbf{k}_{c}-\mathbf{k}_{v}\right|$ ). The intensity of the wave-vector excitation $(\Delta k \neq 0)$ is stronger than that of the excitation between the bands with the same wave vector $(\Delta k=0)$, and has a peak around $\Delta k \sim 4 \mathrm{~nm}^{-1}$. This is because the present wave-vector excitation is directly induced by the large components of wave vectors inherent in the ONF, which is localized in a nanometer-sized region [15]. Therefore, as well as the absorption, stimulated emission accompanying the change in the wave vector would be possible by use of the ONF.

Figure 7(b) shows that the ONF yields a moderate intensity at $\Delta k=0$, which is also attributed to the wave-vector excitation. This is because the $\Delta k \neq 0$ excitation for the primitive cell is included in the absorption at $\Delta k=0$ for the orthorhombic cell due to the band folding [30]. Indeed, the far-field excitation yields a fairly small absorption at $1.6 \mathrm{eV}$.

From Fig. 7(a), it is further found that the ONF excitation causes a large number of transitions, which result in the high absorption intensity. These transitions originate from the complicated band structure of the realistic system. As shown in Fig. 7(a), there are many band states with different energy surfaces along the wave vector $\mathbf{k}$. Thus, numerous combinations of valence- and conduction-band orbitals have an energy gap equal to the energy of a photon. Figure 6(b) shows the absorption intensities of the ONF

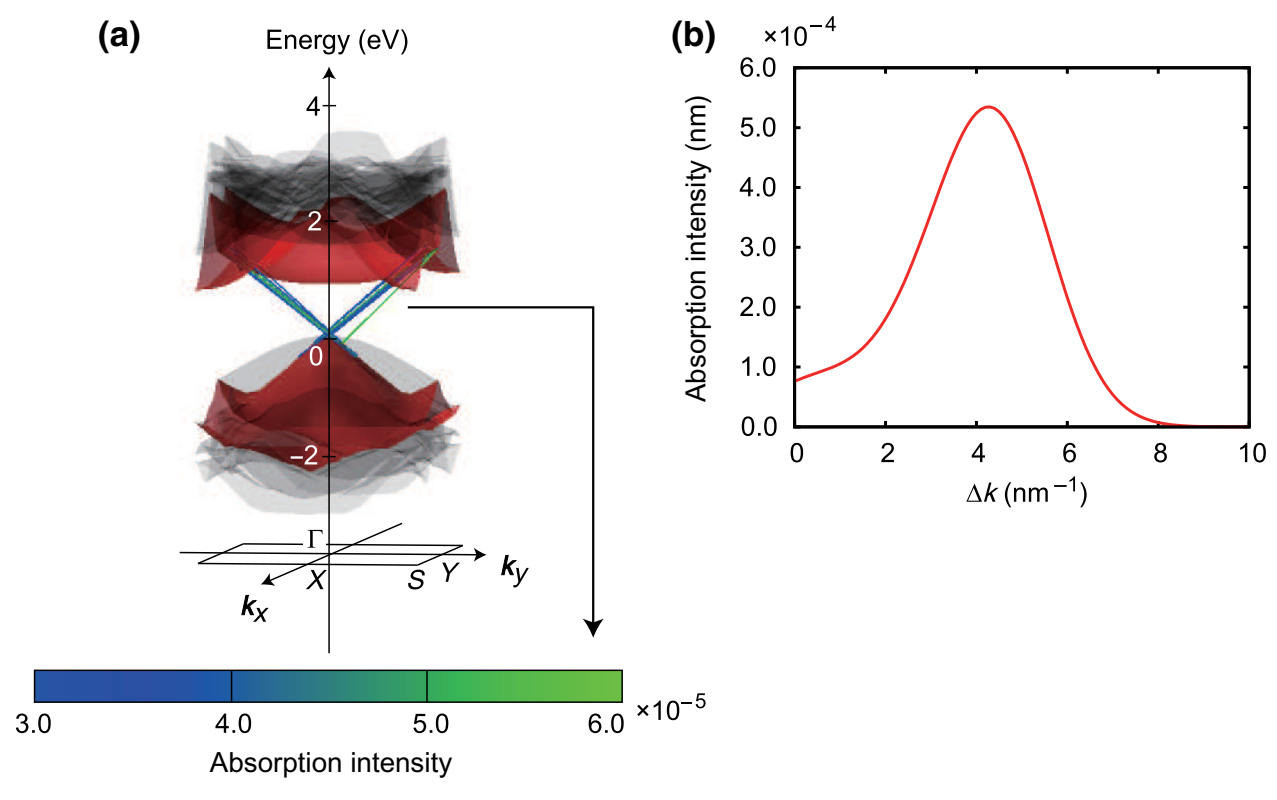

FIG. 7. (a) Map of absorption intensities between the secondhighest valence band and the lowest conduction band (highlighted in red) in the ONF excitation with energy of $1.6 \mathrm{eV}$. Colored bars depict interband excitation paths, and the excitation intensities are shown in the bottom calibrations. (b) Dependence of the absorption intensity on the variation in the wave vector $(\Delta k)$ for the excitation shown in (a). 
and FF excitations, where the intensity of the FF excitation is given as the value per surface area of $2 \times 2 \mathrm{~nm}^{2}$ for comparison (see also Sec. II B). When the photon energy is lower than $2.4 \mathrm{eV}$, the intensity of the FF excitation is a few orders of magnitude lower than that of the ONF excitation; that is, the ONF is capable of enhancing the intensity even if the photon energy is slightly higher than the direct band-gap energy (approximately $2.0 \mathrm{eV}$ ). When the photon energy is greater than $3 \mathrm{eV}$, the intensity of the FF excitation is stronger than that of the ONF excitation. This is because the number of transitions induced by the FF also increases as the photon energy increases, while the net intensity of the ONF localized in space is slightly lower than that of the FF. In other words, the intensity enhancement in the low-energy range is attributed not to the electric field enhancement that is frequently discussed in the plasmonics research field but to the large components of wave vectors inherent in the ONF.

In a model system such as a one-dimensional potentialwell model, the number of transitions remains almost unchanged by the ONF excitation [15]. This is because there are severely limited transitions whose energy matches with the incident laser energy when the band structure is simplified. In other words, the wave-vector excitation cannot be strongly induced for model systems with a simplified band structure. Therefore, to deal with a realistic band structure is very important to discuss the intensity of the wave-vector excitation induced by the ONF. On the basis of the first-principles results, it is demonstrated that the ONF is capable of inducing strong excitation in silicon at a lower energy than its direct band-gap energy.

\section{CONCLUSION}

In conclusion, we perform first-principles calculations of the ONF excitation of silicon, which is a typical material with an indirect-band-gap structure. The absorption edge of the dielectric function of the hydrogen-terminated (111) surface is at $2.1 \mathrm{eV}$, while the edge for the ONF excitation decreases to $1.6 \mathrm{eV}$ because of the direct wavevector excitation. It is clearly shown that the absorption edge of silicon can be shifted to lower photon energy, originating from the ONF excitation. Furthermore, the absorption of the ONF excitation is a few orders of magnitude larger than that of the FF excitation when the energy is lower than $2.4 \mathrm{eV}$. Previous theoretical studies on simplified model systems did not well demonstrate that the wave-vector excitation induced by the ONF has a strong absorption intensity. However, we here elucidate that the wave-vector excitation is strongly induced in the realistic silicon system. This is because of a large number of transitions in the realistic system with a much-morecomplex band structure than that of a simplified model. The principal drawback of using silicon for optical devices due to the indirect-band-gap structure is nearly overcome owing to the insights obtained in this study, demonstrating that direct wave-vector excitation can be realized, while mature industrial techniques for processing silicon to fabricate electronic devices can also be used to develop optical devices. Thus, our first-principles results pave the way for advancement in designing alternative optical devices made of conventional silicon semiconductors.

\section{ACKNOWLEDGMENTS}

This research was supported by JSPS KAKENHI (Grants No. 17H03101, No. 18H05157, and No. 18H01470), MEXT as a social and scientific priority issue ("Creation of new functional devices and highperformance materials to support next-generation industries") to be tackled by using the post-K computer (ID hp170074, hp170250), the JSPS Core-to-Core Program, A. Advanced Research Networks, Research Foundation for Opto-Science and Technology, and JST-CREST under Grant No. JP-MJCR16N5. The research partly used computational resources of Oakforest-PACS provided by the Multidisciplinary Cooperative Research Program in the Center for Computational Sciences, University of Tsukuba. The computations were also partly performed at the Research Center for Computational Science, Okazaki, Japan.

[1] H. A. Atwater, and A. Polman, Plasmonics for improved photovoltaic devices, Nat. Mater. 9, 205 (2010).

[2] N. O. Länk, R. V. Verre, P. Johansson, and M. Käll, Largescale silicon nanophotonic metasurfaces with polarization independent near-perfect absorption, Nano Lett. 17, 3054 (2017).

[3] C.-Y. Yang, J.-H. Yang, Z.-Y. Yang, Z.-X. Zhou, M.-G. Sun, V. E. Babicheva, and K.-P. Chen, Nonradiating silicon nanoantenna metasurfaces as narrowband absorbers, ACS Photonics 5, 2596 (2018).

[4] H. R. Stuart, and D. G. Hall, Absorption enhancement in silicon-on-insulator waveguides using metal island films, Appl. Phys. Lett. 69, 2327 (1996).

[5] D. M. Schaadt, B. Feng, and E. T. Yu, Enhanced semiconductor optical absorption via surface plasmon excitation in metal nanoparticles, Appl. Phys. Lett. 86, 063106 (2005).

[6] C. Hägglund, M. Zäch, and B. Kasemo, Enhanced charge carrier generation in dye sensitized solar cells by nanoparticle plasmons, Appl. Phys. Lett. 92, 013113 (2008).

[7] F. J. Beck, A. Polman, and K. R. Catchpole, Tunable light trapping for solar cells using localized surface plasmons, J. Appl. Phys. 105, 114310 (2009).

[8] B. P. Rand, P. Peumans, and S. R. Forrest, Long-range absorption enhancement in organic tandem thin-film solar cells containing silver nanoclusters, J. Appl. Phys. 96, 7519 (2004).

[9] A. J. Morfa, K. L. Rowlen, T. H. Reilly, M. J. Romero, and J. van de Lagemaat, Plasmon-enhanced solar energy 
conversion in organic bulk heterojunction photovoltaics, Appl. Phys. Lett. 92, 013504 (2008).

[10] S. S. Kim, S. I. Na, J. Jo, D. Y. Kim, and Y. C. Nah, Plasmon enhanced performance of organic solar cells using electrodeposited Ag nanoparticles, Appl. Phys. Lett. 93, 073307 (2008).

[11] R. B. Konda, R. Mundle, H. Mustafa, O. Bamiduro, A. K. Pradhan, U. N. Roy, Y. Cui, and A. Burger, Surface plasmon excitation via $\mathrm{Au}$ nanoparticles in $\mathrm{n}-\mathrm{CdSe} / \mathrm{p}-\mathrm{Si}$ heterojunction diodes, Appl. Phys. Lett. 91, 191111 (2007).

[12] D. H. Lee, J. Y. Kwon, S. Maldonado, A. Tuteja, and A. Boukai, Extreme light absorption by multiple plasmonic layers on upgraded metallurgical grade silicon solar cells, Nano Lett. 14, 1961 (2014).

[13] X. Chen, J. Fang, X. Zhang, Y. Zhao, and M. Gu, Optical/electrical integrated design of core-shell aluminumbased plasmonic nanostructures for record-breaking efficiency enhancements in photovoltaic devices, ACS Photonics 4, 2102 (2017).

[14] M. Kirkengen, and J. Bergli, Direct generation of charge carriers in c-Si solar cells due to embedded nanoparticles, J. Appl. Phys. 102, 093713 (2007).

[15] M. Yamaguchi, and K. Nobusada, Indirect interband transition induced by optical near fields with large wave numbers, Phys. Rev. B 93, 195111 (2016).

[16] M. Noda, K. Ishimura, K. Nobusada, K. Yabana, and T. Boku, Massively-parallel electron dynamics calculations in real-time and real-space: toward applications to nanostructures of more than ten-nanometers in size, J. Comput. Phys. 265, 145 (2014).

[17] M. Noda, M. Yamaguchi, and K. Nobusada, Second harmonic excitation of acetylene by the optical near field generated in a porous material, J. Phys. Chem. C 121, 11687 (2017).

[18] K. Iida, M. Noda, and K. Nobusada, Development of theoretical approach for describing electronic properties of hetero-interface systems under applied bias voltage, J. Chem. Phys. 146, 084706 (2017).

[19] J. P. Perdew, and A. Zunger, Self-interaction correction to density-functional approximations for many-electron systems, Phys. Rev. B 23, 5048 (1981).
[20] J. P. Perdew, K. Burke, and Y. Wang, Generalized gradient approximation for the exchange-correlation hole of a manyelectron system, Phys. Rev. B 54, 16533 (1996).

[21] N. Troullier, and J. L. Martins, Efficient pseudopotentials for plane-wave calculations, Phys. Rev. B 43, 1993 (1991).

[22] L. Kleinman, and D. M. Bylander, Efficacious Form for Model Pseudopotentials, Phys. Rev. Lett. 48, 1425 (1982).

[23] K. Yabana, Y. Shinohara, T. Otobe, J.-I. Iwata, and G. F. Bertsch, in Advances in Multi-photon Processes and Spectroscopy, edited by S. H. Lin, A. A. Villaeys, and Y. Fujimura (World Scientific, 2008), Vol. 18, p. 209.

[24] R. M. Martin, Electronic Structure: Basic Theory and Practical Methods (Cambridge University Press, Cambridge, 2004).

[25] N. W. Ashcroft, and N. D. Mermin, Solid State Physics (Holt, Rinehart and Winston, New York, 1976).

[26] P. Giannozzi, QUANTUM ESPRESSO: a modular and open-source software project for quantum simulations of materials, J. Phys.: Condens. Matter 21, 395502 (2009).

[27] C. A. Ullrich, Time-Dependent Density-Functional Theory: Concepts and Applications (OUP, Oxford, 2011).

[28] S.-Y. Yang, H. Yang, E. Derunova, S. S. Parkin, B. Yan, and M. N. Ali, Symmetry demanded topological nodal-line, Adv. Phys.: X 3, 1414631 (2018).

[29] S. Ciraci, R. Butz, E. M. Oellig, and H. Wagner, Chemisorption of hydrogen on the Si(100) surface: Monohydride and dihydride phases, Phys. Rev. B 30, 711 (1984).

[30] See Supplemental Material at http://link.aps.org/supplemen tal/10.1103/PhysRevApplied.11.044053 for the details of the time-dependent occupation number and excitation intensity, the dependence of the excitation intensity on the transition energy, the absorptions between the highest valence band and the lowest conduction band, and the interband excitation at $\Delta \mathrm{k}=0$.

[31] C. Kittel, Introduction to Solid State Physics (8th ed.John Wiley, New York, 2004).

[32] N. Ashcroft, N. Mermin, and D. Wei, Solid State Physics: Revised Edition (Cengage Learning Asia, Singapore, 2016). 